\title{
The History and Legacy of BATSE
}

\author{
Gerald J. (Jerry) Fishman ${ }^{1}$ \\ ZP12, NASA-Marshall Space Flight Center, Huntsville, AL, 35812 USA \\ E-mail: jerry.fishman@nasa.gov
}

The BATSE experiment on the Compton Gamma-ray Observatory was the first large detector system specifically designed for the study of gamma-ray bursts. The eight large-area detectors allowed full-sky coverage and were optimized to operate in the energy region of the peak emission of most GRBs. BATSE provided detailed observations of the temporal and spectral characteristics of large samples of GRBs, and it was the first experiment to provide rapid notifications of the coarse location of many them. It also provided strong evidence for the cosmological distances to GRBs through the observation of the sky distribution and intensity distribution of numerous GRBs. The large number of GRBs observed with the high- sensitivity BATSE detectors continues to provide a database of GRB spectral and temporal properties in the primary energy range of GRB emission that will likely not be exceeded for at least another decade. The origin and development of the BATSE experiment, some highlights from the mission and its continuing legacy are described in this paper. 


\section{The Origin of BATSE}

Soon after the announcement of the discovery of GRBs by the Los Alamos Group with the Vela satellites ${ }^{1}$, it was realized that balloon flight observations of them were possible. An extrapolation of an expected $-3 / 2$ power law intensity distribution of them, expected for a homogeneous, three-dimensional distribution of GRB sources to lower intensities would yield a GRB rate of $\sim$ several per day, as observed with a balloon-borne detector system with an effective area of $\sim 1 \mathrm{~m}^{2}$, during a balloon flight of reasonable duration. Using the cosmic ray research facilities and personnel kindly made available by T. Parnell at the NASA-Marshall Space Flight Center (MSFC), I decided to begin a modest balloon flight program aimed at observing GRBs that were considerably weaker that those observed previously.

Large-area crystal scintillation detectors were fabricated using cheap, scrap pieces of sodium iodide scintillator crystals immersed in clear mineral oil ${ }^{2,3}$. These hermetically sealed detectors had a crystal thickness of $\sim 2 \mathrm{~cm}$, which have a high detection efficiency for GRB radiation up to a few hundred $\mathrm{keV}$. Two balloon flights with a total duration of 28 hours were carried out in 1975 and 1977 from Palestine, Texas using an array of these type of detectors. These observations resulted in an observed rate of GRBs that was well below that expected for a homogeneous, three-dimentional distribution of GRBs ${ }^{4}$. Balloon flights in 1980 and 1982 used arrays of more expensive, single-crystal detectors with higher light output, similar to that shown in Figure 1. This resulted in better measurements of the rate of weak gamma ray bursts ${ }^{5}$. These later detectors were prototypes of the detectors that were eventually used on BATSE.

\section{The BATSE Proposal}

NASA Headquarters issued an Announcement of Opportunity (AO) in 1977 soliciting proposals for instruments for a large Gamma-Ray Observatory (GRO), originally scheduled for launch in 1985. Initially, our balloon group in Huntsville had not planned to submit a proposal, but at the suggestion of T. Cline and with the encouragement of T. Parnell, one was submitted by the Huntsville gamma-ray group, with G. Fishman as the Principal Investigator, and C. Meegan and T. Parnell as Co-Investigators.

The objectives of the experiment were to observe the sky distribution and the intensity distribution of GRBs, along with the spectral and high-time-resolution properties of a large number of them. In addition, this experiment would provide a "trigger signal" to the other GRO instruments, so that their wide-field detectors could also respond to a gamma-ray burst detected by our instrument. This was a key element of our proposal; it was to be a "service" to the other, larger experiments that had GRB observations as a secondary objective of their proposals. These experiments were not optimized for GRB observations and they did not have full-sky coverage. This strategy was suggested by $\mathrm{T}$. Cline.

The principal design philosophy for the BATSE detectors was to maximize the collecting area and monitor the entire sky for GRBs for a large number of bursts, while providing a rough location for them. High time resolution for the data was also important. Background reduction and good energy resolution were of less importance for the instrument. BATSE was the 
smallest, lightest, had the lowest data rate and was the least expensive of the instruments that were selected for GRO in 1978.

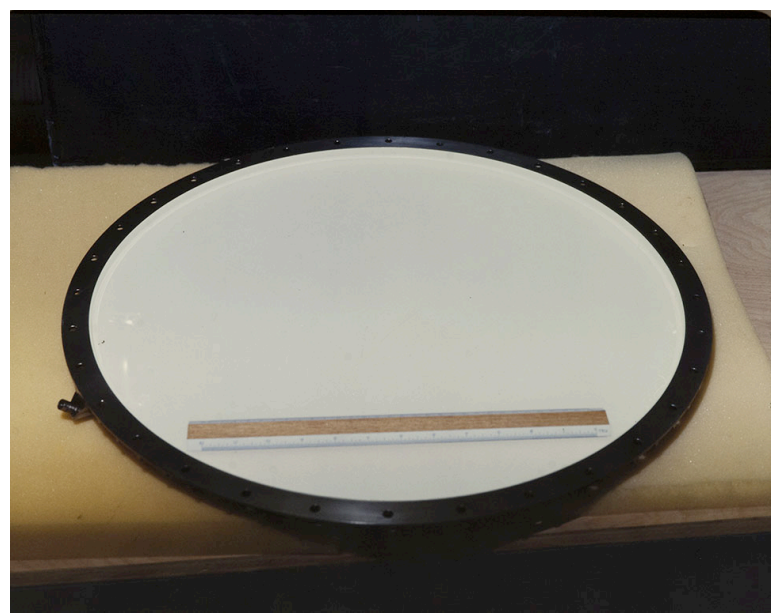

Figure 1: The scintillation detector element of a Large Area Detector (LAD) for BATSE. The design and dimensions are similar to those manufactured for medical diagnostic purposes in devices known as Anger cameras. It consists of a circular, hermetically-sealed disc of thalliumactivated sodium iodide, $\mathrm{NaI}(\mathrm{Tl})$, optically-coupled to a thick, fused silica optical window. The crystal had a thickness of $1.27 \mathrm{~cm}$ and a surface area of $2025 \mathrm{~cm}^{2}$. Details of the design, development and testing of the BATSE flight system are contained in the comprehensive publication by J. Horack ${ }^{6}$.

\section{Developing GRO and Launch into Orbit}

In 1980, it was determined that one of the five instruments selected for GRO had to be removed. This difficult decision arose from a combination of limitations of GRO for mass and volume, and also for cost considerations. A review panel was convened to provide input to NASA Headquarters, which made the final decision. In 1981, it was determined that the Gamma-ray Spectrometer Experiment (GRSE) would not be part of the GRO spacecraft. The final configuration of the spacecraft is shown in Figure 2, along with one of the BATSE detector modules.

After the elimination of GRSE from GRO, it was recognized that the spacecraft would not have the capability for wide-field, high-spectral resolution observations of GRBs. At that time, spectral lines from GRBs were reportedly observed by several groups; these were deemed to be high priority observations for the GRO mission. These reported lines were believed to arise from positron annihilation, cyclotron line production, and/or redshifted nuclear excitation lines in the gravitational field of Galactic neutron stars, at that time presumed to be the source of GRBs. An appeal was made to NASA Headquarters by a group of GRB theorists to include a capability for these observations on GRO. NASA agreed, and in response, the BATSE team (with additional investigators) submitted a proposal to include an additional detector in each of the eight BATSE modules, smaller than the LAD, but thicker and with better energy resolution. It would cover a broader energy range (both higher and lower) than the LAD. These detectors were termed the Spectroscopy Detectors (SDs). This proposal was accepted by NASA 
Headquarters. The majority of the design, development and testing of the BATSE instrumentation was performed at NASA-MSFC.

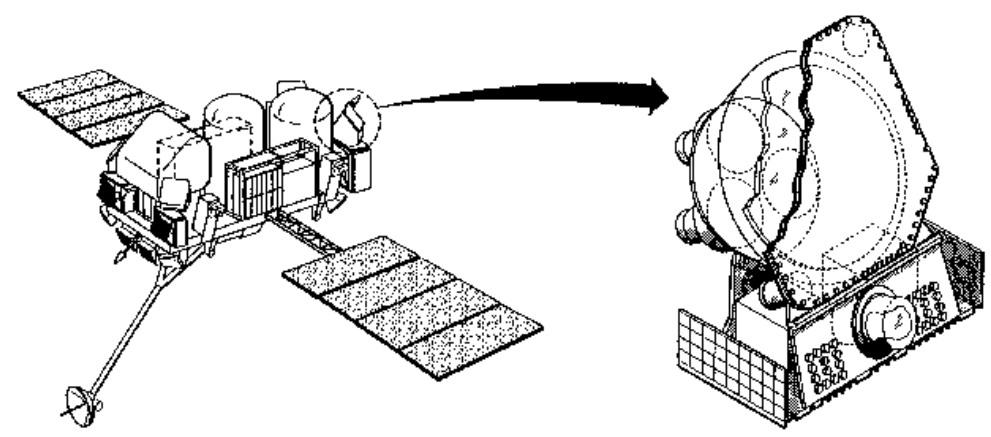

Figure 2: Left: The on-orbit configuration of the CGRO spacecraft with eight BATSE detector modules at the corners of the spacecraft. Each module had nearly a clear forward field-of-view. Right: One of the detector modules, showing a cut-away view of a large area detector (LAD) and a smaller spectroscopy detector (SD).

Although the BATSE SDs had considerably better energy resolution than the LADs, their sensitivity was much less than that of the LADs in the energy region, $\sim 30 \mathrm{keV}$ to $\sim 600 \mathrm{keV}$. However, at lower and at higher energies, the SDs had greater sensitive area than the LADs for the following reasons: Below $30 \mathrm{keV}$, the LAD efficiency dropped sharply due to absorbing material in front of the detector and above $600 \mathrm{keV}$, the LAD efficiency decreased due to the transparency of the relatively thin $\mathrm{NaI}$ detector.

Most of the BATSE development activities occurred during the years 1983-1988. In late 1988, the BATSE flight system was delivered to the facilities of the spacecraft contractor, TRW Inc., in Redondo Beach, California. BATSE underwent two years of integration and testing with the GRO spacecraft. It was then shipped to the Kennedy Space Flight Center (KSC) for integration and testing with the Space Shuttle Atlantis. GRO was launched and deployed into an orbit at $450 \mathrm{~km}$ in April 1991. When it became operational about a month later, it was renamed the Compton Gamma-ray Observatory (CGRO).

\section{Primary GRB Results and the Legacy of BATSE}

Before the end of its first year in orbit, the observed isotropic sky distribution together with the intensity distribution of GRBs, as observed with BATSE, showed with high significance that their origin was unlike that of any known Galactic distribution ${ }^{7}$ and they were they not associated with any known extragalactic objects or regions. Over the next few years, as the statistical measurements of these distributions became more accurate, more workers in the GRB field were abandoning models of GRBs originating from Galactic neutron stars. The most likely explanation was that the GRBs originated from cosmological distances. The final BATSE sky distribution of GRBs is shown in Figure 3. The definitive recognition of their cosmological distances had to wait for more precise and rapid GRB locations, the follow-up xray and optical observations of GRB afterglows, and the redshift measurements of their host galaxies. These were made possible by BeppoSAX observations in 1997 and 1998. 


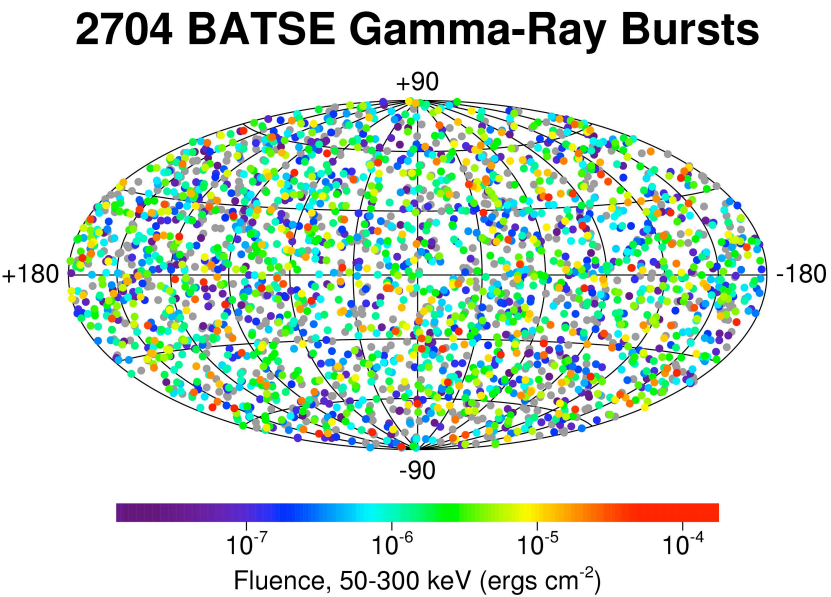

Figure 3: The BATSE sky distribution of GRBs observed over nine years of observation by BATSE-CGRO, plotted in Galactic coordinates. This distribution has not been corrected for sky exposure. The color of each GRB corresponds to the indicated fluence of the burst.

Data from BATSE triggered $\mathrm{GRBs}^{8}$ are available online (http://heasarc.gsfc.nasa.gov/). Nearly a thousand of papers have been published describing the observed temporal and spectral properties of GRBs using these BATSE data. Limits to gamma-ray lines from GRBs using the BATSE spectroscopy detectors were found to be below those of line fluxes reported previously'. In an effort to find additional GRBs that were not part of the standard BATSE GRB catalogs, several investigators compiled catalogs of un-triggered BATSE GRBs.

There are generally accepted to be two classes of GRBs, short and long; the usual dividing line between long and short GRBs is $\sim 2 \mathrm{~s}$, although there is significant overlap between these classes. Prior to BATSE, it had been suspected that the shorter GRBs had harder spectra than the longer ones. BATSE data showed the definitive separation between the short/hard and the long/soft classes with very good statistics ${ }^{10}$.

In 1993, a system known as BACODINE (for BATSE Coordinates Distribution Network) was implemented at GSFC. This system was made possible due to the need for real-time data from the spacecraft caused by the deterioration of the CGRO tape recorders. The design and implementation of BACODINE was the work of Scott Barthelmy ${ }^{11}$. It used the near real-time BATSE data to compute a coarse GRB location. Automated GRB location messages were made available to users for rapid GRB follow-up observations. Even though these locations were usually of the order of several square degrees, they were useful for wide-field optical cameras. Several optical systems were constructed specifically for this purpose. One of these robotic systems was able to observe the optical emission from the intense burst GRB990123 while the burst was in progress ${ }^{12}$. As other spacecraft with GRB capabilities were placed into orbit, their data were also distributed to the GRB community over this same network. The BACODINE system evolved into a more general system, the GRB Coordinates Network (GCN), which distributes data from many spacecraft and is used by hundreds of observers. It has become an invaluable service for the GRB community. 


\section{Conclusion}

The large number of GRBs observed with BATSE, afforded by its sensitive area and long duration in orbit, allowed observations of the temporal and spectral properties of GRBs in more detail than those previously. Observations with BATSE afforded an unsurpassed study of many of the fine points of the gamma-ray emission in the energy region where the major fraction of energy is emitted during the prompt phase. These details, along with the large number of GRBs observed with a single instrument will likely will not be exceeded for many years ${ }^{13}$.

\section{References}

[1] R. W. Klebesadel, I. B. Strong, and R. A. Olson, "Observations of Gamma-Ray Bursts of Cosmic Origin", ApJ 182 (1973) L85.

[2] G. J. Fishman, "Radiation Detectors Using Multiple Scintillation Crystal Pieces", U.S. Patent $\# 3,835,325$ (1974).

[3] G. J. Fishman and R. W. Austin, "Large-area, Multi-crystal NaI(Tl) Detectors for X-ray and Gamma-ray Astronomy”, Nucl. Inst. and Meth. 140 (1976) 193.

[4] G. J. Fishman, C. A. Meegan, J. W. Watts, Jr., and J. H. Derrickson, "New Limits on GammaRay Bursts", ApJ 223 (1978) L13.

[5] C. A. Meegan, G. J. Fishman, and R. B. Wilson, "The Frequency of Weak Gamma-ray Bursts", ApJ 291 (1985) 479.

[6] J. M. Horack, "Development of the Burst and Transient Source Experiment", NASA Reference Publication 1268 (1991).

[7] C. A. Meegan, G. J. Fishman, R. B. Wilson, J. M. Horack, et al., "Spatial Distribution of Gamma-ray Bursts Observed by BATSE”, Nature 355 (1992) 143.

[8] W.S. Paciesas, C. A. Meegan, G. N. Pendleton, et al., "The Fourth BATSE Gamma-Ray Burst Catalog (Revised)", ApJS 122 (1999) 465.

[9] M. S. Briggs, D. L. Band, R. D. Preece, et al., "Analysis of Line Candidates in Gamma-Ray Bursts Observed by BATSE”, ApLett\&Comm 39 (1999) 237.

[10] C. Kouveliotou, C. A. Meegan, G. J. Fishman, N. P. Bhat, et al., "Identification of Two Classes of Gamma-ray Bursts", ApJ 413 (1993) L101

[11] S. D. Barthelmy, P. Butterworth, T. L. Cline, et al., "BACODINE, the Real-Time BATSE Gamma-Ray Burst Coordinates Distribution Network”, Ap\&SpSci 231 (1995) 235.

[12] C. Akerlof, R. Balsano, S. Barthelmy, et al., "Observation of contemporaneous optical radiation from a $\gamma$-ray burst”, Nature 398 (1999) 400

[13] G. J. Fishman and C. A. Meegan, “The BATSE Era”, Chapter 3, in Gamma-ray Bursts, C. Kouveliotou, et al., Eds., Cambridge Univ. Press (2012), in press. 